\title{
Laplace transforms related to excursions of a one-dimensional diffusion
}

\author{
JIM PITMAN $^{1}$ and MARC YOR ${ }^{2}$ \\ ${ }^{1}$ Department of Statistics, University of California, 367 Evans Hall 3860, Berkeley, CA 94720- \\ 3860,USA.e-mail:pitman@stat.berkeley.edu \\ ${ }^{2}$ Université Pierre et Marie Curie, Laboratoire de Probabilités, 4 Place Jussieu - Tour 56, \\ 75252 Paris Cedex 05, France
}

Various known expressions in terms of hyperbolic functions for the Laplace transforms of random times related to one-dimensional Brownian motion are derived in a unified way by excursion theory and extended to one-dimensional diffusions.

Keywords: Brownion motion; hyperbolic function; local time

\section{Introduction}

It is well known that the Laplace transforms of many random times derived from a onedimensional Brownian motion (BM) admit simple expressions in terms of hyperbolic functions. This paper offers a unified approach to these results, and presents their generalizations for a one-dimensional diffusion, using Itô's (1971) excursion theory. See Jeanblanc et al. (1997) for a survey of related results involving the Feynman-Kac formula for the distribution of an additive functional of BM, and see Borodin and Salminen (1996) for a vast array of fomulae for the distribution of functionals of a one-dimensional diffusion.

Section 2 presents the basic univariate formulae in a table, together with commentary and proofs. Section 3 shows how the univariate formulae can be combined with independence results from excursion theory to obtain various multivariate Laplace transforms. In the case of BM, these results have been applied to process control and stockmarket prices by Taylor (1975), and to the asymptotic distribution of windings of planar BM by Pitman and Yor (1986).

\section{Univariate transforms}

Let $I$ be a subinterval of the real line. Let $\left(P^{x}, x \in I\right)$ govern $X=\left(X_{t}, t \geqslant 0\right)$ as a nonsingular diffusion on $I$. For background and precise definitions, see Itô and McKean (1965), Rogers and Williams (1987) or Borodin and Salminen (1996). Assume for simplicity that $X$ is recurrent. Let $0, x \in I$ with $0 \leqslant x$. Let $\lambda \geqslant 0$. In each row of Table 1 , the left-hand entry is 
the $P^{x}$ expectation of some functional of the diffusion path, mostly for $x=0$. The middle entry gives a general expression for this expectation in terms of three basic functions: $g_{\lambda}(x, 0)$, the $\lambda$-potential density with respect to the speed measure; $s(x)$, the scale function; $\phi_{\lambda}(x)$, the $P^{0}$ Laplace transform of $T_{x}=\inf \left\{t: X_{t}=x\right\}$. These basic functions are interpreted probabilistically by Rows 1,3 and 5 of Table 1 . Analytic expressions for these functions, in terms of the semigroup or generator of $X$, are standard.

Explicit formulae for the basic functions are known for many diffusions, including Bessel and Ornstein-Uhlenbeck processes (Borodin and Salminen 1996). In particular, the third column of the table gives formulae derived from the second column in the case when $X$ is a reflecting Brownian motion $(\mathrm{RBM})$ on $I=[0, \infty)$, in terms of hyperbolic functions of $\theta x$, where $\theta=(2 \lambda)^{1 / 2}$. All the formulae in the third column were obtained by Knight (1969), who also inverted most of these transforms. For $X$ a standard BM on $I=(-\infty, \infty)$ the formulae in Table 1 apply for all $x \geqslant 0$ with

$$
g_{\lambda}(x, 0)=\theta^{-1} \mathrm{e}^{-\theta x}, \quad s(x)=2 x, \quad \phi_{\lambda}(x)=\mathrm{e}^{-\theta x},
$$

where $\theta=(2 \lambda)^{1 / 2}$. Then $s_{\lambda}(x)=\theta^{-1}\left(1-\mathrm{e}^{-2 \theta x}\right)$.

The following commentary introduces the notation of Table 1, line by line, and indicates proofs of the formulae by application of Itô's excursion theory.

Table 1. Some basic Laplace transforms

\begin{tabular}{llll}
\hline Row & Probabilistic quantity & $\begin{array}{l}\text { General expression } \\
\text { for } 0 \leqslant x\end{array}$ & $\begin{array}{l}\text { Expression for RBM } \\
\text { with } \theta=(2 \lambda)^{1 / 2}\end{array}$ \\
\hline 1 & $P^{x}\left(L_{W_{\lambda}}\right)$ & $g_{\lambda}(x, 0)$ & $\frac{\exp (-\theta x)}{\theta}$ \\
2 & $P^{0}\left(\mathrm{e}^{-\lambda \tau^{\prime}}\right)$ & $\exp \left(\frac{-\ell}{g_{\lambda}(0,0)}\right)$ & $\exp (-\ell \theta)$ \\
3 & $P^{0}\left(L_{T_{x}}\right)$ & $s(x)$ & $\exp \left(\frac{-\ell}{x}\right)$ \\
4 & $P^{0}\left(M_{\tau_{\zeta}} \leqslant x\right)$ & $\exp \left(\frac{-\ell}{s(x)}\right)$ & $\frac{1}{\cosh (\theta x)}$ \\
5 & $P^{0}\left(\mathrm{e}^{-\lambda T_{x}}\right)$ & $\phi_{\lambda}(x)$ & $\frac{\tanh (\theta x)}{\theta}$ \\
6 & $P^{0}\left(L_{T_{x} \wedge W_{\lambda}}\right)$ & $s_{\lambda}(x)=g_{\lambda}(0,0)-\phi_{\lambda}(x) g_{\lambda}(x, 0)$ & $\exp \{-\ell \theta \operatorname{coth}(\theta x)\}$ \\
7 & $P^{0}\left\{\mathrm{e}^{-\lambda \tau_{\prime}} 1\left(M_{\tau}, \leqslant x\right)\right\}$ & $\exp \left(\frac{-\ell}{s_{\lambda}(x)}\right)$ & $\frac{\tanh (\theta x)}{\theta x}$ \\
8 & $P^{0}\left(\mathrm{e}^{-\lambda G_{x}}\right)$ & $\frac{s_{\lambda}(x)}{s_{(x)}}$ & $\frac{\theta x}{\sinh (\theta x)}$ \\
9 & $P^{0}\left\{\mathrm{e}^{-\lambda\left(T_{x}-G_{x}\right)}\right)$ & $\frac{\phi_{\lambda}(x) s(x)}{s_{\lambda}(x)}$ & \\
\hline
\end{tabular}




\subsection{Row 1}

Let $L=\left(L_{t}, t \geqslant 0\right)$ be a local time process of $X$ at 0 , and let $W_{\lambda}$ be exponentially distributed with rate $\lambda$, independent of $X$. This row identifies the potential density probabilistically as

$$
g_{\lambda}(x, 0)=P^{x}\left(L_{W_{\lambda}}\right)=P^{x} \int_{0}^{\infty} \lambda \mathrm{e}^{-\lambda t} L_{t} \mathrm{~d} t=P^{x} \int_{0}^{\infty} \mathrm{e}^{-\lambda t} \mathrm{~d} L_{t}=c \int_{0}^{\infty} \mathrm{e}^{-\lambda t} p(t, x, 0) \mathrm{d} t,
$$

where $p(t, x, y)=p(t, y, x)=P^{x}\left(X_{t} \in \mathrm{d} y\right) /(m \mathrm{~d} y)$ is the jointly continuous transition density of $X$ relative to the speed measure $m$, and $c$ is a constant depending on the normalization of local time and conventions regarding constant factors in the definition of the scale function and speed measure of $X$. To be precise, it is supposed that $L_{t}=L_{t}^{0}$ where the process of local times $\left(L_{t}^{y}, t \geqslant 0, y \in I\right)$ is such that

$$
\int_{0}^{t} f\left(X_{s}\right) \mathrm{d} s=c \int_{I} f(y) L_{t}^{y} m \mathrm{~d} y
$$

almost surely for every non-negative Borel function $f$. In the third column, for $X$ a RBM, say $X=|B|$ where $B$ is a BM, and also for $X=B$, we take $L$ to be the occupation density of $B$ at 0 relative to Lebesgue measure. Then Lévy's equivalence holds; $L_{t}$ and $\left|B_{t}\right|$ have the same $P^{0}$ distribution.

\subsection{Row 2}

Let $\left(\tau_{\ell}, \ell \geqslant 0\right)$ be the inverse of $L$. The general expression for the Laplace transform of $\tau_{\ell}$ is well known for $L$, the local time process of $X$ at 0 for any recurrent point 0 of a strong Markov process $X$. This formula follows immediately from the probabilistic definition of $g_{\lambda}(0,0)$ in Row 1 , by Itô's excursion theory. Let $P^{0}$ govern a Poisson point process $N$ on $(0, \infty)$ with rate $\lambda$, independent of $X$, and mark each excursion of $X$ away from 0 by the times of points of $N$ during the excursion, if any. Then, as explained by Greenwood and Pitman (1980) and Rogers and Williams (1987, Section VI.53), one obtains a homogeneous Poisson point process of marked excursions on the local time scale. (In the case when $X$ spends positive Lebesgue time at 0 , this process must also count marks between excursions.) Let $W_{\lambda}$ be the time of the first point of $N$. Then $L_{W_{\lambda}}$ is the time of the first marked excursion on the local time scale; so $L_{W_{\lambda}}$ has exponential distribution with rate $1 / P\left(L_{W_{\lambda}}\right)=1 / g_{\lambda}(0,0)$. Thus

$$
P^{0}\left(\mathrm{e}^{-\lambda \tau_{\ell}}\right)=P^{0}\left(W_{\lambda}>\tau_{\ell}\right)=P^{0}\left(L_{W_{\lambda}}>\ell\right)=\exp \left(\frac{-\ell}{g_{\lambda}(0,0)}\right) .
$$

Analysis of this formula, together with Krein's theory of strings, allowed Knight (1981) and Kotani and Watanabe (1982) to characterize the Lévy measures of the process of inverse local times $\left(\tau_{\ell}, \ell \geqslant 0\right)$. In particular, these Lévy measures are absolutely continuous with respect to Lebesgue measure on $(0, \infty)$, and the densities are Laplace transforms. See also Section 6 of Pitman (1996). 


\subsection{Row 3}

This row defines $s(x)$ for $x>0$. Note that $1 / s(x)$ is the rate per unit local time of excursions from 0 that reach $x$. So, by the Poisson character of the excursion process, and the strong Markov property of $X$, for $0<x<y$, given that an excursion reaches $x$, the chance that it reaches $y$ is

$$
P^{x}\left(T_{y}<T_{0}\right)=\frac{1 / s(y)}{1 / s(x)}=\frac{s(x)}{s(y)} .
$$

That is to say, the function $s(x)$ serves as a scale function for $X$ on the interval $[0, \infty]$, with $s(0)=0$.

\subsection{Row 4}

Here $M_{t}=\max _{0 \leqslant s \leqslant t} X_{s}$. This is implied by Row 3 and the Poisson character of excursions on the local time scale, just as Row 1 implied Row 2.

\subsection{Row 5}

This row defines $\phi_{\lambda}(x)$. The evaluation of $\phi_{\lambda}(x)$ for RBM is made by the following wellknown argument: for $\theta=(2 \lambda)^{1 / 2}$, apply the optional sampling theorem to the martingale $\cosh \left(\theta\left|B_{t}\right|\right) \exp (-\lambda t)$ which is the average of the two martingales $\exp \left( \pm \theta B_{t}-\lambda t\right)$.

\subsection{Row 6}

This row defines a new function

$$
\begin{aligned}
s_{\lambda}(x) & :=P^{0}\left(L_{T_{x} \wedge W_{\lambda}}\right) \\
& =P^{0}\left(L_{W_{\lambda}}\right)-P^{0}\left\{\left(L_{W_{\lambda}}-L_{T_{x}}\right) 1\left(T_{x}<W_{\lambda}\right)\right\} \\
& =g_{\lambda}(0,0)-\phi_{\lambda}(x) g_{\lambda}(x, 0)
\end{aligned}
$$

by application of the strong Markov property of $X$ at time $T_{x}$, and the definitions of Rows 1 and 3. Substituting the formulae of Rows 1 and 3 for RBM gives the expression $s_{\lambda}(x)=\theta^{-1} \tanh (\theta x)$ for RBM.

\subsection{Row 7}

This is implied by Row 6, just as Row 1 implies Row 2, and Row 3 implies Row 4. In terms of the Poisson point process of marked excursions, Row 6 shows that $1 / s_{\lambda}(x)$ is the rate of excursions that either reach $x$ or are marked. The left-hand and middle entries of Row 7 show two different ways of computing the probability of no such excursions up to local time $\ell$. 


\subsection{Row 8}

Here $G_{x}$ is the last zero of $X$ before time $T_{x}$. Consider the first excursion that either reaches $x$ or is marked. Compute the probability that this excursion reaches $x$, first by conditioning on $G_{x}$, then from the ratio of Poisson rates $\{1 / s(x)\} /\left\{1 / s_{\lambda}(x)\right\}$, to see that this probability is given by both the left and central entries of Row 8 .

\subsection{Row 9}

The Poisson character of the excursion process implies that $G_{x}$ and $T_{x}-G_{x}$ are independent (last exit decomposition). So Row 9 follows from Rows 5 and 8. For $X$ a BM or RBM, the result is implicit in Williams' description of the process $\left(X_{G_{x}+t}, 0 \leqslant t \leqslant T_{x}-G_{x}\right)$ as a BES(3) process started at 0 and run till it first hits $x$ (Williams 1974, equation (67.2) of Chapter II). More generally, if the upper end point of the basic interval $I$ on which $X$ is defined is $b$ say, Williams' results show that the $P^{0}$ distribution of $\left(X_{G_{x}+t}, 0 \leqslant t \leqslant T_{x}-G_{x}\right)$ is identical with the $\hat{P}^{0}$ distribution of $\left(X_{t}, 0 \leqslant t \leqslant T_{x}\right)$ where the family of diffusion laws $\left(\hat{P}^{x}, x \in[0, b)\right.$ ) conditions $X$ to hit $b$ before 0 (the Doob $h$ transform of $X$ for $h(x)=s(x)$ ). So Row 9 implies an expression for the $\hat{P}^{0}$ Laplace transform of $T_{x}$ :

$$
\hat{P}^{0}\left\{\exp \left(-\lambda T_{x}\right)\right\}=\frac{\phi_{\lambda}(x) s(x)}{s_{\lambda}(x)} .
$$

The generator $\hat{A}$ for this conditioned diffusion is $\hat{A}=s^{-1} A s$, where $A$ is the generator of $X$. Using the standard fact (Itô and McKean 1965, Section 4.6) that $1 / \phi_{\lambda}(x)$ is a solution of $A f=\lambda f$, it is easy enough to check that the inverse of the right side of (1) solves $\hat{A} f=\lambda f$ off 0 . A more careful discussion of boundary behaviour is required to make this observation into an analytic proof of (1). See Jeanblanc et al. (1997) for related results.

\section{Multivariate transforms}

As noted by Knight $(1969,1978)$, for each $x>0$ the Poisson character of the excursion process implies that $\left(X_{t}, 0 \leqslant t \leqslant G_{x}\right)$ given $L_{T_{x}}=\ell$ has the same distribution as $\left(X_{t}, 0 \leqslant t \leqslant \tau_{\ell}\right)$ given $\left(M_{\tau_{\ell}}<x\right)$. Combined with Rows 4 and 7 of Table 1 this implies that

$$
P^{0}\left\{\exp \left(-\lambda G_{x}\right) \mid L_{T_{x}}=\ell\right\}=P^{0}\left\{\exp \left(-\lambda \tau_{\ell}\right) \mid M_{\tau_{\ell}}<x\right\}=\exp \left\{-\ell \xi_{\lambda}(x)\right\},
$$

where

$$
\xi_{\lambda}(x):=\frac{1}{s_{\lambda}(x)}-\frac{1}{s(x)} \stackrel{\text { BBM }}{=} \theta \operatorname{coth}(\theta x)-\frac{1}{x}
$$

and the notation $\stackrel{\text { RBM }}{=}$ means equality in the case when $X$ is RBM, with $\theta=(2 \lambda)^{1 / 2}$. This result for RBM is due to Knight (1969). Combine (2) and (3) with the fact that $L_{T_{x}}$ has exponential distribution with rate $1 / s(x)$ to obtain 


$$
P^{0}\left\{\exp \left(-\alpha L_{T_{x}}-\lambda G_{x}\right)\right\}=\frac{s_{\lambda}(x)}{s(x)\left\{1+\alpha s_{\lambda}(x)\right\}} \stackrel{\mathrm{RBM}}{=} \frac{1}{\theta x \operatorname{coth}(\theta x)+\alpha x} .
$$

Using the independence of $\left(L_{T_{x}}, G_{x}\right)$ and $T_{x}-G_{x}$, and Row 9, this implies that

$$
P^{0}\left\{\exp \left(-\alpha L_{T_{x}}-\lambda T_{x}\right)\right\}=\frac{\phi_{\lambda}(x)}{1+\alpha s_{\lambda}(x)} \stackrel{\text { RBM }}{=} \frac{\theta}{\theta \cosh (\theta x)+\alpha \sinh (\theta x)} .
$$

Williams (1976) obtained this formula for RBM and used it to deduce the closely related formulae of Taylor (1975).

Consider now the occupation times

$$
A_{t}^{+}:=\int_{0}^{t} \mathrm{~d} s 1\left(X_{s}>0\right), \quad A_{t}^{-}:=\int_{0}^{t} \mathrm{~d} s 1\left(X_{s} \leqslant 0\right) .
$$

As a preliminary for computation of the $P^{0}$ joint Laplace transform of $A_{T_{x}}^{+}, A_{T_{x}}^{-}$and $L_{T_{x}}$, observe from (2) and the independence of positive and negative excursions that there is the identity

$$
P^{0}\left\{\exp \left(-\lambda A_{\tau_{\ell}}^{+}\right) M_{\tau_{\ell}}<x\right\} P^{0}\left\{\exp \left(-\lambda A_{\tau_{\ell}}^{-}\right)\right\}=\exp \left\{-\ell \xi_{\lambda}(x)\right\}
$$

Now write $A_{\tau_{\ell}}^{+}=B_{\ell}(x)+C_{\ell}(x)$ where $B_{\ell}(x)$ is the total length of those positive excursions before time $\tau_{\ell}$ which fail to reach $x$, and $C_{\ell}(x)$ is the total length of those positive excursions before time $\tau_{\ell}$ which do reach $x$. By another application of the Poisson character of the excursion process, $B /(x)$ and $C_{/}(x)$ are independent. Since the events $\left(M_{\tau_{\ell}}<x\right)$ and $\left(C_{\ell}(x)=0\right)$ are identical, and $B /(x) \downarrow 0$ as $x \downarrow 0$, it follows that

$$
P^{0}\left\{\exp \left(-\lambda A_{\tau_{\ell}}^{+}\right) \mid M_{\tau_{\ell}}<x\right\}=P^{0}\left[\exp \left\{-\lambda B_{\ell}(x)\right\}\right] \uparrow 1 \text { as } x \downarrow 0
$$

and hence from (7)

$$
P^{0}\left\{\exp \left(-\lambda A_{\tau_{\ell}}^{-}\right)\right\}=\exp \left\{-\ell \xi_{\lambda}(0+)\right\}
$$

where

$$
\xi_{\lambda}(0+):=\lim _{x \downarrow 0} \xi_{\lambda}(x) \stackrel{\mathrm{BM}}{=} \frac{\theta}{2}
$$

and $\stackrel{\text { BM }}{=}$ means equality in the case when $X$ is BM, with $\theta=(2 \lambda)^{1 / 2}$. Now (7) gives

$$
P^{0}\left\{\exp \left(-\lambda A_{\tau_{\ell}}^{+}\right) \mid M_{\tau_{\ell}}<x\right\}=\exp \left[-\ell\left\{\xi_{\lambda}(x)-\xi_{\lambda}(0+)\right\}\right]
$$

and (8), (10) and the independence of positive and negative excursions combine to yield

$$
P^{0}\left\{\exp \left(-\lambda A_{\tau_{\ell}}^{+}-\mu A_{\tau_{\ell}}^{-}\right) \mid M_{\tau_{\ell}}<x\right\}=\exp \left[-\ell\left\{\xi_{\lambda}(x)-\xi_{\lambda}(0+)+\xi_{\mu}(0+)\right\}\right] .
$$

Conditioning on $L_{T_{x}}=\ell$, as before, and then integrating out $\ell$ yields the formula

$$
P^{0}\left\{\exp \left(-\alpha L_{T_{x}}-\lambda A_{G_{x}}^{+}-\mu A_{G_{x}}^{-}\right)\right\}=\frac{s_{\lambda}(x) / s(x)}{1+\left\{\alpha+\xi_{\mu}(0+)-\xi_{\lambda}(0+)\right\} s_{\lambda}(x)} .
$$

Since $A_{T_{x}}^{-}=A_{G_{x}}^{-}$and $A_{T_{x}}^{+}=A_{G_{x}}^{+}+\left(T_{x}-G_{x}\right)$ where $T_{x}-G_{x}$ is independent of ( $L_{T_{x}}$, $A_{G_{x}}^{-}, A_{G_{x}}^{+}$) by the last exit decomposition, (12) combined with Row 9 of Table 1 yields finally 


$$
\begin{aligned}
P^{0}\left\{\exp \left(-\alpha L_{T_{x}}-\lambda A_{T_{x}}^{+}-\mu A_{T_{x}}^{-}\right)\right\} & =\frac{\phi_{\lambda}(x)}{1+\left\{\alpha+\xi_{\mu}(0+)-\xi_{\lambda}(0+)\right\} s_{\lambda}(x)} \\
& \stackrel{\text { BM }}{=} \frac{\theta}{\theta \cosh (\theta x)+\left\{2 \alpha+(2 \mu)^{1 / 2} \sinh (\theta x)\right.}
\end{aligned}
$$

Pitman and Yor (1986, Proof of Theorem 4.2) found this formula for BM by martingale calculus and applied it to the asymptotic distribution of windings of a planar BM.

\section{Acknowledgement}

This research was supported in part by National Science Foundation Grant DMS 94-04345.

\section{References}

Borodin, A. and Salminen, P. (1996) Handbook of Brownian Motion-Facts and Formulae. Boston, MA: Birkhäuser.

Greenwood, P. and Pitman, J. (1980) Fluctuation identities for Lévy processes and splitting at the maximum. Adv. Appl. Probab., 12, 893-902.

Itô, K. (1971) Poisson point processes attached to Markov processes. Proceedings of the Sixth Berkeley Symposium on Mathematical Statistics and Probability, Vol. 3, pp. 225-240. Berkeley, CA: University of California Press.

Itô, K. and McKean, H. (1965) Diffusion Processes and their Sample Paths. Berlin: Springer-Verlag. Jeanblanc, M., Pitman, J. and Yor, M. (1997) The Feynman-Kac formula and decomposition of Brownian paths. Comput. Appl. Math., 16, 27-52.

Knight, F. (1969) Brownian local times and taboo processes. Trans. Amer. Math. Soc., 143, 173-185.

Knight, F. (1978) On sojourn times of killed Brownian motion. In Séminaire de Probabilités XII, pp. 428-445. Lecture Notes in Math. 649. Berlin: Springer-Verlag.

Knight, F. (1981) Characterization of the Lévy measure of inverse local times of gap diffusions. Seminar on Stochastic Processes, 1981, pp. 53-78. Boston, MA: Birkhäuser.

Kotani, S. and Watanabe, S. (1982) Krein's spectral theory of strings and generalized diffusion processes. In M. Fukushima (ed.) Functional Analysis in Markov Processes, pp. 235-249. Lecture Notes in Math. 923. Berlin: Springer-Verlag.

Pitman, J. (1996) Cyclically stationary Brownian local time processes. Probab. Theory Related Fields, 106, 299-329.

Pitman, J. and Yor, M. (1986) Asymptotic laws of planar Brownian motion. Ann. Probab., 14, 733-779. Rogers, L. and Williams, D. (1987) Diffusions, Markov Processes and Martingales. New York: Wiley. Taylor, H.M. (1975) A stopped Brownian motion formula. Ann. Probab., 3, 234-246.

Williams, D. (1974) Path decomposition and continuity of local time for one dimensional diffusions I. Proc. London Math. Soc., Third Ser., 28, 738-768.

Williams, D. (1976) On a stopped Brownian motion formula of H.M. Taylor. In Séminaire de Probabilités X, pp. 235-239. Lecture Notes in Math. 511. Berlin: Springer-Verlag.

Received August 1996 and revised July 1997 